\title{
Neurogenic Thoracic Outlet Syndrome: Causing by Cervical Rib
}

\author{
Patpiya Sirasaporn
}

\begin{abstract}
Neurogenic thoracic outlet syndrome is an uncommon disease. It may causes by cervical rib, scar tissues which compress to structure at thoracic outlet area such as subclavian artery, subclavian vein and brachial plexus. The symptoms which are caused by compressing these structures are pale, swelling, numbness, weakness and pain. The author reports a case of twenty one years old female who complained mass at left supraclavicular area and numbness at left hand and forearm. Her electrodiagnostic study diagnosed neurogenic thoracic outlet syndrome. Partial left cervical rib resection was done in this case. After operation for 4 months, the symptoms were improved. Therefore, the diagnosis of this syndrome is important that helps clinicians to correctly plan of treatment.
\end{abstract}

Keywords: Neurogenic thoracic outlet syndrome; Cervical rib; Electrodiagnostic study

\section{Introduction}

Thoracic outlet syndrome is characterized by the presence of compressing to structure at thoracic outlet such as subclavian artery, subclavian vein and brachial plexus. The causes are cervical rib, abnormal posture, trauma, scalene muscle hypertrophy and fibrous band [1]. Cervical rib is a congenital anomaly which is originated from enlargement of the transverse process of $\mathrm{C} 7$. This anomaly occurs $1 \%$ of the population but induces the symptoms about $5 \%$ [2]. The symptoms are depended on compressed structure such as pale, swell-

Manuscript accepted for publication January 14, 2014

Department of Physical Medicine and Rehabilitation, Faculty of Medicine, Khon Kaen University, Khon Kaen 40002, Thailand. Email: spatpiya@kku.ac.th

doi: http://dx.doi.org/10.14740/jmc1669w ing, edema, numbness, weakness and pain [3]. As a result of various symptoms, the diagnosis of this syndrome is very complex [4].

\section{Case Report}

Case twenty one years old female complained a hard palpable mass at left supraclavicular area for 1 year. She underwent tissue biopsy that was diagnosed reactive lymphoid tissue. She felt numbness at left hand and forearm. Sometimes, she felt pain radiating from left shoulder to little finger. She denied other abnormalities. There was no previous history of trauma or systemic disease.

Physical examination revealed a firm mass at left supraclavicular area and no muscle atrophy. There were normal brachial and radial pulsations. Motor power was grade 5 all. Decreased pinprink sensation and temperature sensation at medial side of left hand were noted. Tinel' sign was positive at left supraclavicular area. Adson's test at left side was negative. The cervical spine radiograph showed bilateral cervical ribs with the left being more angulation (Fig. 1).

Nerve conduction studies (NCS) and needle electromyography were conducted. NCS showed prolong latency, normal amplitude and normal nerve conduction velocity at left ulnar motor and sensory nerves while left median motor, lat-

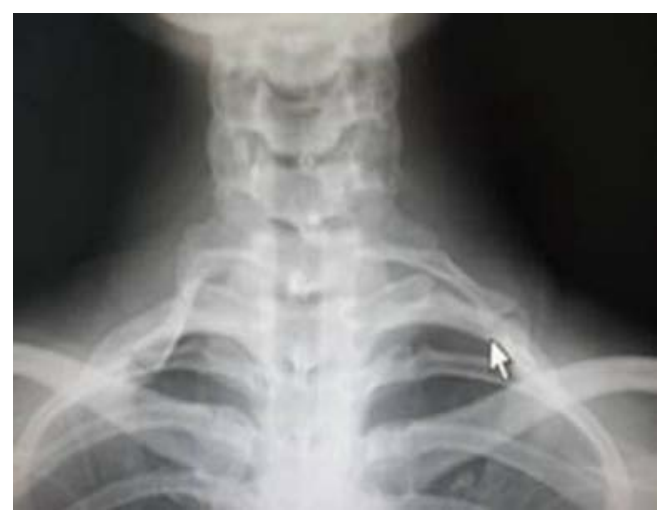

Figure 1. It shows bilateral cervical ribs. The arrow points out left cervical rib. 
Table 1. Motor Nerve Conduction Studies

\begin{tabular}{lllll}
\hline Nerve & Stimulation & Latency (ms) & Amplitude (mV) & Velocity (m/s) \\
\hline \multirow{2}{*}{ Left ulnar } & wrist & $4.6^{*}$ & 10.4 & \\
& Below elbow & 7.6 & 10.0 & 63 \\
Left median & wrist & 3.8 & 13.0 & 56 \\
& elbow & 7.0 & 13.0 & \\
\hline
\end{tabular}

* prolong latency.

Table 2. Sensory Nerve Conduction Studies

\begin{tabular}{lllll}
\hline Nerve & Stimulation & Latency (ms) & Amplitude (mV) & Velocity (m/s) \\
\hline Left lateral antebrachial cutaneous & forearm & 2.6 & 5 \\
Left medial antebrachial cutaneous & forearm & 2.6 & 7 \\
Left ulnar & wrist & $3.9^{*}$ & 37 & 61 \\
& elbow & 6.5 & 33 & 61 \\
\hline
\end{tabular}

* prolong latency.

eral antebrachial cutaneous and medial antebrachial cutaneous nerves were within normal range (Table1, 2). In needle electromyography showed normal study at left deltoid, biceps, brachioradialis and dorsal interosseous muscles. This result was summarized to sign of focal demyelin involvement at left ulnar nerve which may caused by neurogenic thoracic outlet syndrome.

Therefore, operation was done under general anesthesia. There was an angulated left cervical rib which was compromised to division of left brachial plexus. Partial left cervical rib resection was undergone by orthopedic surgeon (Fig. 2). There was no operation related complications. Four months after operation, she had no pain and felt better in sensation at left hand and forearm.

\section{Discussion}

The neurogenic thoracic outlet syndrome most commonly involves the lower cervical nerves of the brachial plexus [5]. The symptoms are most frequently impact the ulnar distribution as this case. The Adson's test is done to mainly assess compression of the subclavian artery which is special test for vasculogenic thoracic outlet syndrome. This report showed neurogenic thoracic outlet syndrome from cervical rib, so the Adson's test was also negative. Due to the relatively uncommon presentation of cervical rib, patients presenting with a neck mass should be thoroughly assessed to rule out any other pathology such as lymphadenopathy and carcinoma

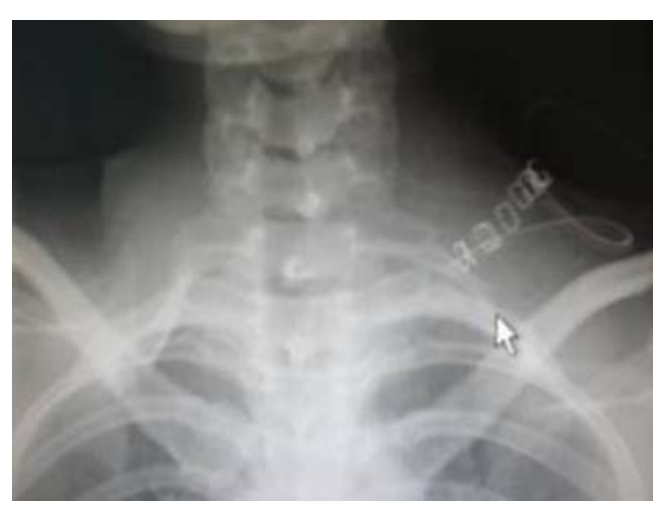

Figure 2. It shows partial left cervical rib resection. 
[6]. Cevical rib is usually asymptomatic and may not require any treatment [7]. However, in this case reveals neck mass with persistent pain and numbness. So, it is an indication for surgery.

\section{Conclusion}

Diagnosis and treatment of thoracic outlet syndrome is not simple routine. Adequate investigations are required. It is important for physician to be careful to detect cause of thoracic outlet syndrome.

\section{Grant Support}

No grant support, No commercial party having a direct financial interest in the results of the research supporting this article has or will confer a benefit upon the authors or upon any organization with which the authors are associated.

\section{References}

1. Luoma A, Nelems B. Thoracic outlet syndrome. Thoracic surgery perspective. Neurosurg Clin N Am. 1991;2(1):187-226.

2. Pollack EW. Surgical anatomy of the thoracic outlet syndrome. Surg Gynecol Obstet. 1980;150(1):97-103.

3. Oates SD, Daley RA. Thoracic outlet syndrome. Hand Clin. 1996;12(4):705-718.

4. Wilbourn AJ. Thoracic outlet syndrome is overdiagnosed. Muscle Nerve. 1999;22(1):130-136; discussion 136-137.

5. Cruz-Martinez A, Arpa J. Electrophysiological assessment in neurogenic thoracic outlet syndrome. Electromyogr Clin Neurophysiol. 2001;41(4):253-256.

6. Leong SC, Karkos PD. A "hard" neck lump. Singapore Med J. 2009;50(4):e141-142.

7. Huang JH, Zager EL. Thoracic outlet syndrome. Neurosurgery. 2004;55(4):897-902; discussion 902-893. 